\title{
Recombination of Aromatic Radicals with Molecular Oxygen
}

\author{
Feng Zhang, ${ }^{1,2,{ }^{*}}$ André Nicolle, ${ }^{2,3}$ Lili Xing, ${ }^{1}$ and Stephen J. Klippenstein ${ }^{2}$
}

\author{
${ }^{1}$ National Synchrotron Radiation Laboratory, University of Science and Technology of China, Hefei, Anhui 230029, P. R. \\ China \\ ${ }^{2}$ Chemical Sciences and Engineering Division, Argonne National Laboratory, Argonne, IL 60439, USA \\ ${ }^{3}$ IFP Energies Nouvelles, 1-4 Avenue de Bois-Préau, 92852 Rueil-Malmaison Cedex, France
}

\section{Corresponding Authors:}

Feng Zhang

National Synchrotron Radiation Laboratory, University of Science and Technology of China, Hefei, Anhui 230027,

P. R. China

Tel: +86-551-63607923

Fax: +86-551-65141078

E-mail: feng2011@ustc.edu.cn

\section{Colloquium:}

\section{REACTION KINETICS}

\section{Word Count (Method 1):}

The total word count (exclusive of title page, abstract) is: $\mathbf{6 1 9 5}$ words

Word Count (was performed from automatic counting function in MS Word plus Figs/Table/References)

\begin{tabular}{|c|c|c|c|c|}
\hline Abstract: & 116 words, not inc & $\mathrm{n}$ word $\mathrm{c}$ & & \\
\hline Main text: & 3955 words & & & \\
\hline References: & 510 words ( 35 refe & & & \\
\hline Tables: & $\mathbf{0}$ words (0 table) & & & \\
\hline Equations: & 30 words ( 2 equat & ngle colu & & \\
\hline Figures: & $\mathbf{1 5 8 4}$ words (8 fig & th captior & & \\
\hline & Figure & Column & Height $/ \mathrm{mm}$ & Word Count \\
\hline & 1 & double & 83 & 411 \\
\hline & 2 & single & 85 & 209 \\
\hline & 3 & single & 49 & 130 \\
\hline & 4 & single & 51 & 134 \\
\hline & 5 & single & 53 & 138 \\
\hline & 6 & single & 52 & 136 \\
\hline & 7 & single & 52 & 136 \\
\hline & 8 & single & 46 & 123 \\
\hline & Captions & & & 167 \\
\hline
\end{tabular}

Total: $\mathbf{6 1 9 5}$ words

Supplementary Material: one supplementary material is available. 


\title{
Recombination of Aromatic Radicals with Molecular Oxygen
}

Feng Zhang, ${ }^{1,2, *}$ André Nicolle, ${ }^{2,3}$ Lili Xing, ${ }^{1}$ and Stephen J. Klippenstein ${ }^{2}$

${ }^{1}$ National Synchrotron Radiation Laboratory, University of Science and Technology of China, Hefei, Anhui 230029, P. R. China

${ }^{2}$ Chemical Sciences and Engineering Division, Argonne National Laboratory, Argonne, IL 60439, USA

${ }^{3}$ IFP Energies Nouvelles, 1 et 4 Avenue de Bois-Préau, 92852 Rueil-Malmaison Cedex, France Institut Carnot IFPEN Transports Energie

\begin{abstract}
:
The addition of molecular oxygen to hydrocarbon radicals yields peroxy radicals (ROO), which are crucial species in both atmospheric and combustion chemistry. For aromatic radicals there is little known about the recombination kinetics, especially for the high temperatures of relevance to combustion. Here, we have employed direct CASPT2 based variable reaction coordinate transition state theory to predict the high pressure recombination rates for four prototypical aromatic hydrocarbon radicals: phenyl, benzyl, 1-naphthyl, and 2-naphthyl. The variation in the predicted rates is discussed in relation to their molecular structure. The predicted rate coefficients are in reasonably satisfactory agreement with the limited experimental data and are expected to find utility in chemical modeling studies of PAH growth and oxidation.
\end{abstract}

Keywords: hydrocarbon peroxy radicals; aromatic radicals; recombination reactions; quantum chemical calculations; variable reaction coordinate transition state theory 


\section{Introduction}

Aromatic radicals, $\mathrm{R}$, are key components in mechanisms for polycyclic aromatic hydrocarbon (PAH) growth and soot formation [1]. They are particularly important in aromatic fuels such as benzene, toluene, xylene, and naphthalene, which are key components of various surrogate fuels. An accurate understanding of their oxidation kinetics is a crucial aspect of combustion modeling $[2,3]$. In the low temperature oxidation environment, ROO chemistry is of direct relevance to the chain reactions that ultimately lead to autoignition. Phenyl, benzyl, and naphthyl radicals are prototypical aromatic radicals, which have received considerable attention in combustion modeling studies (see, e.g, $[2,4,5])$. Such modeling studies have generally estimated the kinetic data for the fuel radical $\mathrm{R}$ $+\mathrm{O}_{2}$ reaction from experience or via extrapolation of relatively low-temperature experimental measurements [6-11].

For phenyl, measurements of Yu et al. [6] over the 297-473 K temperature range (at 20-80 Torr) suggest that the $\mathrm{O}_{2}$ recombination rate constant decreases with temperature, while Schaugg et al.'s measurements showed a positive temperature dependence at slightly higher temperatures (418-815 K at 0.5 Torr) [9]. For benzyl, both Hoyermann et al. [10] and Nelson et al. [11] observed no temperature dependence over the 243-373 K range (at pressures of 1-3 and 3-15 Torr, respectively), while Fenter et al. indicated a significant decline with increasing temperature at analogous temperatures (298-398 K) (and higher pressures of 20-760 Torr) [7]. Only Park et al. have measured the rate constant for 2-naphthyl, finding a modest decrease over the 299-444 K temperature range [8] (at 40 Torr). These discrepancies in the observed temperature behavior add to the uncertainty in any attempted extrapolations to combustion temperatures.

There is also discordancy in recent theoretical predictions of the temperature dependence for aromatic radicals $+\mathrm{O}_{2}$ recombination kinetics. The canonical variational transition state theory 
(CVTST) calculations of da Silva et al. [12] predict that the rate coefficient for phenyl $+\mathrm{O}_{2}$ should decrease by about a factor of 4 over the 300-2000 K temperature range. In contrast, the very recent variable reaction coordinate transition state theory (VRC-TST) [13] calculations of Kislov et al. [14] predict that for phenyl, 1- and 2-naphthyl radicals the $\mathrm{O}_{2}$ recombination rate constant should rise over the 418-2500 $\mathrm{K}$ range, in qualitative accord with the observation of a positive temperature dependence by Schaugg et al. [9, 14].

In this work, the $\mathrm{O}_{2}$ recombination kinetics was studied for four representative aromatic hydrocarbon radicals: phenyl, benzyl, 1- and 2-naphthyl radicals. The radical characteristics of triplet $\mathrm{O}_{2}$ result in a barrierless minimum energy path (MEP) for its addition to radicals $[15,16]$. Prior applications of the VRC-TST approach, such as the recent application to the closely related $\mathrm{C}_{2} \mathrm{H}_{3}+$ $\mathrm{O}_{2}$ addition reaction [15], have amply demonstrated its ability to accurately predict the rate coefficients for such barrierless radical-radical reactions.

Hence, the present analysis employs VRC-TST as in the work of Kislov et al. [14], but proceeds beyond it in a variety of ways. Their approximate representation of the transitional mode interaction potential employing qualitative bending anisotropies is replaced here with direct CASPT2 determinations. In addition, more accurate estimates of the MEP energies are obtained through multireference based evaluations of the spin-splitting combined with coupled-cluster based evaluations for the high spin quartet state, as in the $\mathrm{C}_{2} \mathrm{H}_{3}+\mathrm{O}_{2}$ study [15]. These improvements should yield significantly more reliable predictions of both the absolute rate constants and their temperature dependence.

\section{Methodology}

\subsection{Kinetic theory}


The key assumption in VRC-TST is the separation of the "conserved" and "translational" modes in the transition state region of the potential $[13,17]$. The conserved modes, which correspond to the vibrational modes of the reacting fragments, are treated as quantized harmonic oscillators. The transitional modes correspond to the remaining modes, which describe the rotational and translational motions of the two reacting fragments. The essential advantage of the VRC-TST approach is its quantitative treatment of the full coupling and anharmonicity of the transitional modes through Monte Carlo integration over coordinates within the classical phase space representation for the reactive flux for a variable set of reaction coordinates.

The variability of the reaction coordinate in VRC-TST is achieved by choosing a set of pivot points whose locations and separations determine the transition state dividing surface. The rate constant is then minimized with respect to both the pivot point locations and separation distances. For the four $\mathrm{R}+\mathrm{O}_{2}$ reactions studied in this work, two sets of dividing surfaces were used. The first set employs center-of-mass $(\mathrm{CoM})$ pivot points, which are appropriate for large separations, while the second set employs orbital centered pivot points, which are appropriate for shorter separations.

A separate 6-point-mesh with a variable grid spacing of 1.0-4.0 au was used for the larger separations (9.0-21.0 au) where the CoM pivot points are used. A 12-point-mesh with finer grids (0.3-1.0 au) was used for the shorter separations (3.8-9.0 au) with orbital centered pivot points. For the orbital centered pivot points, their displacement from the bonding atoms was varied from 0.5 to $1.5 \mathrm{au}$, with a step size of $0.5 \mathrm{au}$. The appropriateness of these mesh sizes is based on past experience for related systems. With the preferred E,J-resolved VRC-TST, which was employed here, the reaction coordinate minimizations are performed for each energy, E, and total angular momentum $\mathrm{J}$. Trajectory calculations indicate that VRC-TST typically overestimate the rate coefficient by about $10-15 \%[18,19]$. Thus, a dynamical correction factor of $(0.85)$ is used for the final rate coefficients. 
These calculations were performed with the VaReCoF code [20] with $8 \%(1 \sigma)$ convergence for the Monte Carlo integrations.

\subsection{Quantum chemical calculations}

The implementation of the VRC-TST approach requires accurate interfragment interaction energies for $\sim 10^{4}$ configurations for each reaction. The VaReCoF program allows for the evaluation of these interactions on-the-fly, with direct calls to ab initio electronic structure methods. The complete active space self-consistent field theory with second-order perturbation (CASPT2) [21] method combined with the cc-pVDZ basis set efficiently yields interaction energies of suitable accuracy $[15,16]$. Here, a 7-electron 5-orbital $(7 \mathrm{e}, 5 \mathrm{o})$ active space was chosen to optimize the MEP for all four $\mathrm{R}+\mathrm{O}_{2}$ reactions. This space correlates with the two pairs of $\mathrm{O}-\mathrm{O} \pi$ and $\pi^{*}$ orbitals, and the radical orbital of $\mathrm{R}$. An ionization potential-electronic affinity (IPEA) shift of 0.25 was introduced into the CASPT2 calculations [22].

The CASPT2/cc-pVDZ energy computed for each sampled configuration is corrected by an orientationally independent factor $\left[\Delta \mathrm{V}_{\text {tot }}\left(\mathrm{R}_{\mathrm{CO}}\right)\right]$, where $\mathrm{R}_{\mathrm{CO}}$ is the bond length for the incipient $\mathrm{CO}$ bond. This correction factor includes both a geometry relaxation correction to account for the use of rigid geometries in the transitional mode sampling $\left(\Delta \mathrm{V}_{\text {relax }}\right)$ and a higher-level correction to account for limitations in the basis set $\left(\Delta \mathrm{V}_{\text {basis }}\right)$ and in the CASPT2 method $\left(\Delta \mathrm{V}_{\text {method }}\right) . \Delta \mathrm{V}_{\text {relax }}$ was defined as the energy difference between the MEPs obtained for rigid and relaxed scanning at the CASPT2(7e,5o)/cc-pVDZ level. $\Delta \mathrm{V}_{\text {basis }}$ is obtained from CBS extrapolation [23] of CASPT2 calculations for the cc-pVTZ and cc-pVQZ bases. The method correction arises from the observation in recent work on $\mathrm{C}_{2} \mathrm{H}_{3}+\mathrm{O}_{2}$ [15] that a more consistent estimate for the doublet state interaction energy may be obtained from the sum of a coupled cluster based estimate of the quartet interaction 
energy and a multireference [CASPT2 or Davidson corrected multirerence configuration interaction (MRCI+Q)] estimate of the doublet-quartet splitting. Note that direct application of CCSD(T) to the doublet state MEP does not yield accurate energies due to the multi-reference nature of the doublet wave function in the transition state region. This method correction is effective because the CCSD(T) method accurately treats the high-spin quartet state, while multi-reference treatments of the spin-splitting are more consistent and reliable than corresponding treatments of the absolute interaction energy. Here, the CCSD(T)/CBS limit for the quartet state interaction energy is obtained from CCSD(T)-F12/VDZ-F12 [24, 25], MP2-F12/VDZ-F12 [26] and MP2-F12/VTZ-F12 evaluations yielding:

$\Delta V_{\text {method }}=\left(V_{C C S D(T)-F 12 / V D Z-F 12}^{4}+V_{M P 2-F 12 / V T Z-F 12}^{4}-V_{M P 2-F 12 / V D Z-F 12}^{4}\right)-V_{C A S P T 2 / C B S}^{4}$

where the superscript " 4 " denotes an energy calculated for the quartet state. For phenyl $+\mathrm{O}_{2}$, the smaller size allowed for additional calculations with larger basis sets, i.e., cc-pVTZ-F12 and cc-pVQZ-F12. In summary, the total correction term $\left(\Delta \mathrm{V}_{\text {tot }}=\Delta \mathrm{V}_{\text {relax }}+\Delta \mathrm{V}_{\text {basis }}+\Delta \mathrm{V}_{\text {method }}\right)$ includes three parts, i.e., a geometry relaxation correction, a basis set correction, and a method correction.

For phenyl and benzyl, we also explored the difference between CASPT2 and MRCI+Q MEPs. For benzyl, we also explored a CASPT2 calculation with a $(13 \mathrm{e}, 11 \mathrm{o})$ active space [which includes the additional $(6 \mathrm{e}, 6 \mathrm{o}) \pi$-space of the C6-ring] in order to improve the treatment of the resonance stabilization of the radical.

Reaction enthalpies for the benzyl- and naphthyl- $+\mathrm{O}_{2}$ reactions were computed at the CCSD(T)-F12/VDZ-F12, MP2-F12/VDZ-F12, and MP2-F12/VTZ-F12 levels. Final single point reaction energies were obtained from the expression

$$
E=E_{C C S D(T)-F 12 / V D Z-F 12}+E_{M P 2-F 12 / V T Z-F 12}-E_{M P 2-F 12 / V D Z-F 12}
$$

The geometries and zero-point energies for these reaction enthalpy evaluations were obtained with 
the B2PLYP-D3 density functional method [27], employing the aug-cc-pVDZ basis for $\mathrm{C}_{6} \mathrm{H}_{5} \mathrm{OO}$ and $\mathrm{C}_{7} \mathrm{H}_{7} \mathrm{OO}$, and the cc-pVDZ basis for $\mathrm{C}_{10} \mathrm{H}_{7} \mathrm{OO}$. These density functional theory (DFT) calculations were performed with the Gaussian program [28]. All of the present CASPT2, CCSD(T)-F12, and MP2-F12 calculations were performed with the Molpro program package [29].

\section{Results and Discussions}

\subsection{Structures and reaction enthalpies}

The B2PLYP-D3 optimized structures and reaction enthalpies at $0 \mathrm{~K}$ for the ROO complexes arising from the addition of $\mathrm{O}_{2}$ to phenyl, benzyl, 1- and 2-naphthyl radicals are illustrated in Fig. 1. The Cartesian coordinates and vibrational frequencies for all optimized structures are provided in the Supplemental Material. There are three distinct ROO adducts for the benzyl radical arising from its various resonance configurations. The $\mathrm{C}_{7} \mathrm{H}_{7} \mathrm{O}_{2}-1$ radical, with $\mathrm{O}_{2}$ added on the methyl branch is 20.2 $\mathrm{kcal} / \mathrm{mol}$ (in the trans conformation) more stable than $\mathrm{C}_{7} \mathrm{H}_{7}+\mathrm{O}_{2}$, while the $\mathrm{C}_{7} \mathrm{H}_{7} \mathrm{O}_{2}-2$ and $\mathrm{C}_{7} \mathrm{H}_{7} \mathrm{O}_{2}-3$ radicals are 5-10 kcal/mol endothermic. Thus, only $\mathrm{C}_{7} \mathrm{H}_{7} \mathrm{O}_{2}-1$ (in the trans conformation) was considered in the calculations of the one-dimensional corrections for the addition reaction. This calculated reaction enthalpy differs significantly from the CBS-QB3 value of $22.3 \mathrm{kcal} / \mathrm{mol}$ from Murakami et al [30]. This discrepancy is not surprising given that the $2 \sigma$ error in CBS-QB3 calculated heats of formation for a large set of small combustion related species is $2.5 \mathrm{kcal} / \mathrm{mol}$ [31]. In contrast, we expect the $2 \sigma$ uncertainty in the present estimate to be $<1 \mathrm{kcal} / \mathrm{mol}$.

In the $\mathrm{C}_{10} \mathrm{H}_{7} \mathrm{OO}$ complexes there are two orientations for the $-\mathrm{OO}$ group, denoted as $\mathrm{C}_{10} \mathrm{H}_{7} \mathrm{OO}-\mathrm{R}$ (right), and $\mathrm{C}_{10} \mathrm{H}_{7} \mathrm{OO}-\mathrm{L}$ (left) [cf., Fig. 1 (c) and (d)]. The steric repulsion arising from the interaction of the $-\mathrm{OO}$ group with the nearest $\mathrm{H}$ atom is more significant in $1-\mathrm{C}_{10} \mathrm{H}_{7} \mathrm{OO}$ than in 2- $\mathrm{C}_{10} \mathrm{H}_{7} \mathrm{OO}$. Indeed, 1- $\mathrm{C}_{10} \mathrm{H}_{7} \mathrm{OO}-\mathrm{R}$ maintains $\mathrm{C}_{\mathrm{s}}$ symmetry, while the OOCC dihedral in 
1- $\mathrm{C}_{10} \mathrm{H}_{7} \mathrm{OO}-\mathrm{L}$ is $\sim 50$ degrees. Even with this torsional rotation in $1-\mathrm{C}_{10} \mathrm{H}_{7} \mathrm{OO}-\mathrm{L}$, the distance from the $\mathrm{O}$ to the nearest $\mathrm{H}$ is still $0.08 \AA$ shorter than in $1-\mathrm{C}_{10} \mathrm{H}_{7} \mathrm{OO}-\mathrm{R}$, implying greater steric hindrance in the former configuration. According to the computed reaction enthalpies, $1-\mathrm{C}_{10} \mathrm{H}_{7} \mathrm{OO}-\mathrm{R}$ is 2.2 $\mathrm{kcal} / \mathrm{mol}$ more stable than $1-\mathrm{C}_{10} \mathrm{H}_{7} \mathrm{OO}-\mathrm{L}$. In contrast, the $-\mathrm{OO}$ orientation has very little effect on the structures or enthalpies for the $\mathrm{O}_{2}$ addition to form $2-\mathrm{C}_{10} \mathrm{H}_{7} \mathrm{OO}$, which is similar to the symmetric $\mathrm{C}_{6} \mathrm{H}_{5} \mathrm{OO}$ structure.

The one-dimensional energy profiles required to evaluate $\Delta \mathrm{V}_{\text {tot }}$ were calculated for both 1- $\mathrm{C}_{10} \mathrm{H}_{7} \mathrm{OO}-\mathrm{R}$ and 1- $\mathrm{C}_{10} \mathrm{H}_{7} \mathrm{OO}-\mathrm{L}$. For simplicity, the VRC-TST calculations for $1-\mathrm{C}_{10} \mathrm{H}_{7}+\mathrm{O}_{2}$ employ the mean value of that from the $1-\mathrm{C}_{10} \mathrm{H}_{7} \mathrm{OO}-\mathrm{R}$ and $1-\mathrm{C}_{10} \mathrm{H}_{7} \mathrm{OO}-\mathrm{L}$ MEPs, which should be acceptable because the corrections are quite similar in the transition state regime even though the overall interactions are quite different. For the formation of $2-\mathrm{C}_{10} \mathrm{H}_{7} \mathrm{OO}$ only the $-\mathrm{R}$ configuration was considered as the energy correction for $2-\mathrm{C}_{10} \mathrm{H}_{7} \mathrm{OO}-\mathrm{L}$ is expected to be nearly identical.

Fig. 1. Optimized ROO complexes and reaction enthalpies at $0 \mathrm{~K}(\mathrm{kcal} / \mathrm{mol}$, in parentheses) for (a) phenyl, (b) benzyl, (c) 1-naphthyl, and (d) 2-naphthyl radicals.

\subsection{Phenyl $+\mathrm{O}_{2}$}

Figure 2(a) illustrates the basis set and method dependence of the calculated MEP for the phenyl $+\mathrm{O}_{2}$ association reaction over $\mathrm{R}_{\mathrm{CO}}$ distances of 2 to $4 \AA$, which roughly spans the range of separations for the variational transition state. The potential energy curves were evaluated for geometries along the CASPT2(7,5)/cc-pVDZ relaxed MEP. The CBS correction is seen to decrease the attractiveness at long-range while increasing it at shorter $\mathrm{R}_{\mathrm{CO}}$. The $\mathrm{M} 1$ method correction [from

Eq. (E1)] has only a minor effect on the CASPT2/CBS MEP for $\mathrm{R}_{\mathrm{CO}}$ values greater than $2.3 \AA$. Although not shown here, employing an expression similar to Eq. (E1), but using cc-pVTZ and 
cc-pVQZ bases, yields a method correction, M2, that is essentially identical to M1.

Figure 2(b) reveals the effect of the method correction on the calculated CBS limit MEP for CASPT2, MRCI, and MRCI+Q calculations. The discrepancy between the CASPT2 and MRCI+Q curves provides some indication of the uncertainty in our predicted MEP curves. Notably, the three solid lines (which include the method correction) are in much better agreement than the three dashed lines (which do not include the method correction). After the method correction, the maximum difference between the MRCI+Q and CASPT2 MEP energies is only $25 \%$.

Fig. 2. MEP energies for the phenyl $+\mathrm{O}_{2}$ reaction. PT2 refers to CASPT2, CI refers to MRCI, while M1 represents the method correction evaluated from Eq. (E1).

Figure 3 illustrates the present VRC-TST predictions for the phenyl $+\mathrm{O}_{2}$ high-pressure recombination rate coefficient. The black solid and dashed lines illustrate our two best results. They include the CBS, relaxation, and M2 method correction and differ only in whether the CBS and method corrections are evaluated with CASPT2 or MRCI+Q calculations. The difference between the two provides some indication of the uncertainty in our predictions. At room temperature, the nearly factor of two difference is due to the strong sensitivity of the predicted rate to the energetics at $\mathrm{R}_{\mathrm{CO}} \sim 2.5 \AA$. Above $1000 \mathrm{~K}$ the two results are in good agreement, differing by less than a factor of 1.35. Unfortunately, it is generally not clear whether the CASPT2 or the MRCI+Q interaction energies should be more accurate. Thus, in comparing with experiment we take the average of these two predictions as our best prediction.

The blue line with circles illustrates the results obtained when all correction terms are ignored. At room temperature this reference result is nearly 3 times larger than the best results. But by $1000 \mathrm{~K}$ the reference and fully corrected results are within about $20 \%$. The uncertainty in our predictions is smaller when these results agree, because the error arising from the 1-dimensional assumptions 
implicit in the corrections should then be less important. When only the basis set and relaxation correction are included (pink solid line) the rate constants are overestimated by up to $30 \%$ compared with those including the method correction.

Fig. 3. VRC-TST predicted recombination rate constants for phenyl $+\mathrm{O}_{2}$.

The computed rate constants show relatively modest temperature dependence, with first a negative $\mathrm{T}$ dependence for $\mathrm{T}<900 \mathrm{~K}$, and then a positive $\mathrm{T}$ dependence for higher $\mathrm{T}$. This minimum in the rate constant is indicative of the delicate balance between entropic and enthalpic effects. For both $\mathrm{T}$ ranges, the transition state is moving to shorter separations with increasing temperature and entropic changes correlate with a negative $\mathrm{T}$ dependence, while enthalpic changes correlate with a positive $\mathrm{T}$ dependence. At low temperature, the transition state lies at large separation, where the MEP has a small slope and the decreasing entropy dominates the $\mathrm{T}$ dependence. At higher $\mathrm{T}$, the transition state lies at shorter separation where the MEP has a larger slope and the enthalpic changes dominate the T dependence.

Our best prediction for phenyl $+\mathrm{O}_{2}$ is compared with previously reported experimental and theoretical rate constants in Fig. 4. The VRC-TST predictions are seen to be in good agreement with cavity ring down (CRD) measurements of Yu et al. [6], Tonokura et al. [32] and of Tanaka et al. [33]. Even the observed negative temperature dependence of Yu et al. [6] is accurately reproduced. In contrast, there is a clear discrepancy between the present VRC-TST predictions and the fast flow reactor observations of Schaugg et al., which show a positive $\mathrm{T}$ dependence over the $\mathrm{T}$ range from 418-815 K [9]. This discrepancy is exacerbated when one notes that their experiments were performed at 0.5 Torr, which should be far into fall-off at the higher temperatures according to our 
sample master equation calculations. Notably, the CRD experiments employed considerably higher pressures (20-760 Torr), with Yu et al. [6] noting that no pressure dependence was observed over the 20-80 Torr presure range.

The VTST results of da Silva et al. [12], which are based on a G3B3 scaled O3LYP/6-31G(d) PES, are in reasonable agreement with the present predictions, differing by a factor of 2 or less. However, they do show stronger negative temperature dependence and fail to predict the slightly positive temperature dependence at high temperature. Very recently, Kislov et al. employed VRC-TST to compute the rate constant over the 418 - $2500 \mathrm{~K}$ temperature range [14]. Their results, which are based on an approximate representation of the transitional mode potential from fits to CASPT2(19,14)//CASSCF(9,9)/aug-cc-pVDZ evaluations along the MEP, are 3.5 times greater than ours at high temperature and show a strong positive temperature dependence. These discrepancies are likely indicative of shortcomings in their PES.

Fig. 4. Comparison between our best prediction and previously reported rate constants for phenyl + $\mathrm{O}_{2}$ recombination.

\subsection{1- and 2-naphthyl $+\mathrm{O}_{2}$}

Figure 5 provides a comparison of the MEPs for $\mathrm{O}_{2}$ adding to 1- and 2-naphthyl. The potential energy has been corrected by all three terms, with Eq. (E1) employed for the method correction. 1- $\mathrm{C}_{10} \mathrm{H}_{7} \mathrm{OO}-\mathrm{L}$ and $1-\mathrm{C}_{10} \mathrm{H}_{7} \mathrm{OO}-\mathrm{R}$ are distinguished by the orientation of the $-\mathrm{OO}$ group, as indicated by Fig. 1 . As expected, the potential energy for $1-\mathrm{C}_{10} \mathrm{H}_{7} \mathrm{OO}-\mathrm{R}$ is very similar to that for $2-\mathrm{C}_{10} \mathrm{H}_{7} \mathrm{OO}$. In contrast, there are considerably greater steric interactions with nearby $\mathrm{H}$ atoms for $1-\mathrm{C}_{10} \mathrm{H}_{7} \mathrm{OO}-\mathrm{L}$, which is up to $2.5 \mathrm{kcal} / \mathrm{mol}$ more repulsive than $1-\mathrm{C}_{10} \mathrm{H}_{7} \mathrm{OO}-\mathrm{R}$ in the $\mathrm{R}_{\mathrm{CO}}$ range of $2-4 \AA$. 
Fig. 5. $\mathrm{MEP}$ for $1-\mathrm{C}_{10} \mathrm{H}_{7}+\mathrm{O}_{2}$ and $2-\mathrm{C}_{10} \mathrm{H}_{7}+\mathrm{O}_{2}$.

The present predictions for the high pressure recombination rates for naphthyl $+\mathrm{O}_{2}$ are illustrated in Fig. 6 together with previously reported data, including for phenyl $+\mathrm{O}_{2}$. The present calculations suggest that the rate constant for adding to 1-naphthyl is about 1.6 times lower than that for adding to phenyl. This is expected on the basis of the increased steric repulsion for 1-naphthyl. In contrast, the predicted rate constant for adding to 2-naphthyl is somewhat greater than that for adding to phenyl. The predicted modest decrease with increasing temperature is in reasonable accord with the experiments of Park et al. [8]. However, the experimental data suggest that $\mathrm{O}_{2}$ addition to 2-naphthyl should actually be slower than to phenyl (by about a factor of 2) for temperatures in the 299-444 K range. The experiments were performed at a pressure of 40 Torr, which is similar to that employed in the CRD work on phenyl $+\mathrm{O}_{2}$. Furthermore, the well depths are nearly identical, while there are more vibrational degrees of freedom in naphthyl. Thus, a significant devaition from the high pressure limit is not expected and the reason for the discordance is not clear. It is perhaps worth noting that the theoretical analysis of Kislov also predicts a modest increase in the rate constant from phenyl to 2-naphthyl. However, their rate predictions are again about 3 times higher than the present ones.

Fig. 6. Comparison between VRC-TST rate constants and previous experimental and theoretical studies for $\mathrm{C}_{6} \mathrm{H}_{5}+\mathrm{O}_{2}$ (black), 1- $\mathrm{C}_{10} \mathrm{H}_{7}+\mathrm{O}_{2}$ (red), and 2- $\mathrm{C}_{10} \mathrm{H}_{7}+\mathrm{O}_{2}$ (blue).

\subsection{Benzyl $+\mathbf{O}_{2}$}

The present kinetic analysis for benzyl radical focuses on the formation of the $\mathrm{C}_{7} \mathrm{H}_{7} \mathrm{O}_{2}-1$ radical 
since additions to the radical site for the two other resonance structures of $\mathrm{C}_{7} \mathrm{H}_{7}$ are endothermic (cf., Fig. 1). Figure 7 illustrates the MEP for formation of $\mathrm{C}_{7} \mathrm{H}_{7} \mathrm{O}_{2}-1$ in a trans conformation at various levels. The cis conformation would yield similar predictions, and is implicitly included in the VRC-TST analysis. Notably, due to the resonance stabilization of the $\mathrm{C}_{7} \mathrm{H}_{7}$ radical, which must be broken before the radical- $\mathrm{O}_{2}$ bond is formed, the MEP is significantly less attractive than it is for phenyl. Indeed, for most methods there is even a saddle point at about $\mathrm{R}_{\mathrm{CO}}=2.3 \AA$.

The MEP curves from the CASPT2(7e,5o), CASPT2(13e,11o), and MRCI+Q calculations are again more consistent after the method correction is included (compare the dispersion of the solid lines with that of the dashed lines). However, there is still considerable variation in the predicted MEP potential curves even after including the method correction. For example, the CASPT2(7e,5o) and CASPT2(13e,11o) predicted saddle points differ by $0.9 \mathrm{kcal} / \mathrm{mol}$. The MRCI+Q method, which might be expected to best handle the additional effects of the ring $\pi$ interactions and the resonance stabilization, provides an MEP that is intermediate in value. In the following we consider the results based on the MRCI+Q MEP to provide our best prediction.

Fig. 7. MEP curves for benzyl $+\mathrm{O}_{2}$ at various levels. PT2(75) refers to the CASPT2(7e,5o) method, PT2(1311) refers to the CASPT2(13e,11o) method, and (CI+Q) refers to MRCI+Q(7e,5o) energies.

Figure 8 presents a comparison of our VRC-TST predictions for the benzyl $+\mathrm{O}_{2}$ high-pressure recombination rate constant with previous experimental measurements [7, 10, 34, 35]. Fenter et al. found the reaction to be independent of pressure in the T range of 298-398 K, for pressures of 20 and 760 Torr [7]. Hoyermann et al. studied this reaction at low temperature (243-373 K) and somewhat lower pressures (0.8-3 Torr) [10], where one might expect modest deviations from the high pressure limit. The two measured rate constants are within a factor of 2, with Fenter et al.'s results showing a 
slightly negative temperature dependence, whereas the data of Hoyermann et al. is temperature independent [7, 10]. Elmaimouni et al. studied this reaction between 393 and $433 \mathrm{~K}$ at 1 Torr [34], showing negative temperature dependence but lower rate coefficients. This negative temperature dependence may be an artifact of the low pressures employed. Nelson et al. reported both temperature and pressure independent rate coefficients in the $\mathrm{T}$ range of $295-372 \mathrm{~K}$ and pressure range of 3-15 Torr [11].

Fig. 8. Comparison of the VRC-TST predicted recombination rate constants and previous experimental measurements for benzyl $+\mathrm{O}_{2}$.

The MRCI+Q based predictions, which are intermediate in value between the two CASPT2 predictions, likely provides a better treatment of the resonance stabilization effects and ring $\pi$ interactions. Thus, we consider the MRCI+Q based predictions, which are in reasonably satisfactory agreement with the experimental data, to provide our best predictions. They show a very modest minimum in the rate constant near $400 \mathrm{~K}$ and the decline from room temperature to $400 \mathrm{~K}$ is in accord with experiment. However, the room temperature prediction is a factor of 2 to 4 lower than the experimental values. Near room temperature the CASPT2(7e,5o) and CASPT2(13e,11o) predictions are about a factor of two higher and lower than the MRCI+Q predictions, respectively. It is not clear to us why the increase in the active space for the CASPT2 calculations results in apparently worse rate predictions. With increasing temperature the discrepancy between these three predictions decreases somewhat. We estimate the uncertainty in our predicted rate constant for this reaction to gradually decrease from about a factor of 3 near room temperature to a factor of 1.5 at $2000 \mathrm{~K}$. 


\section{Conclusions}

The high pressure recombination kinetics for $\mathrm{O}_{2}$ adding to a series of prototypical aromatic hydrocarbon radicals were studied with direct CASPT2 based VRC-TST theory. The predictions for phenyl, benzyl, 1- and 2-naphthyl radicals over the 300 to $2500 \mathrm{~K}$ temperature range are accurately reproduced by the modified Arrhenius expressions reported in the Supplementary material. The predicted kinetics for $\mathrm{C}_{6} \mathrm{H}_{5}, 1-\mathrm{C}_{10} \mathrm{H}_{7}$, and 2- $\mathrm{C}_{10} \mathrm{H}_{7}$ are fairly similar, with the rates being lowest for $1-\mathrm{C}_{10} \mathrm{H}_{7}$ due to a modest steric effect. In contrast, the resonantly-stabilized character of the benzyl radical leads to much lower reaction enthalpies and rate coefficients.

For the $\mathrm{O}_{2}$ addition to $\mathrm{C}_{6} \mathrm{H}_{5}, 1-\mathrm{C}_{10} \mathrm{H}_{7}$, and $2-\mathrm{C}_{10} \mathrm{H}_{7}$, the calculations predict a modest negative temperature dependence near room temperature, then show a minimum near $700 \mathrm{~K}$, and finally rise by up to a factor of 2 by $2500 \mathrm{~K}$. The predicted temperature dependence for the rate constants is in excellent agreement with many of the experimental observations, which are generally limited to temperatures of $500 \mathrm{~K}$ and lower. The predicted magnitudes are in satisfactory agreement with experiment, with maximum discrepancies within 50\%. The resonantly-stabilized benzyl radical is more difficult to treat accurately, especially near room temperature where the predictions are very sensitive to the predicted saddle point energy. Nevertheless, our best predictions for benzyl are still in reasonable agreement with experiment. The present systematic kinetic studies for the oxidation of these four typical aromatic radicals enhances our understanding of the structure-activity relationship for such reactions, and should help to improve the rate rules for $\mathrm{R}+\mathrm{O}_{2}$ reactions.

\section{Acknowledgements}

This material is based in part on work at Argonne supported by the U.S. Department of Energy, Office of Science, Office of Basic Energy Sciences, Division of Chemical Sciences, Geosciences, and Biosciences under Contract No. DE-AC02-06CH11357. This work is also financially supported 
by National Natural Science Foundation of China under grants 21303173 and 51376170 . We gratefully acknowledge numerous constructive discussions with Yuri Georgievskii and Lawrence B. Harding.

\section{References}

[1] H. Richter, J.B. Howard, Prog. Energy Combust. Sci. 26 (2000) 565-608.

[2] F. Battin-Leclerc, Prog. Energy Combust. Sci. 34 (2008) 440-498.

[3] J. Zádor, C.A. Taatjes, R.X. Fernandes, Prog. Energy Combust. Sci. 37 (2011) 371-421.

[4] W. Yuan, Y. Li, P. Dagaut, J. Yang, F. Qi, Combust. Flame 162 (2015) 22-40.

[5] C. Saggese, A. Frassoldati, A. Cuoci, T. Faravelli, E. Ranzi, Combust. Flame 160 (2013) 1168-1190.

[6] T. Yu, M.C. Lin, J. Am. Chem. Soc. 116 (1994) 9571-9576.

[7] F.F. Fenter, B. Nozière, F. Caralp, R. Lesclaux, Int. J. Chem. Kinet. 26 (1994) 171-189.

[8] J. Park, Z.F. Xu, M.C. Lin, J. Phys. Chem. A 113 (2009) 5348-5354.

[9] J. Schaugg, R.S. Tranter, H.-H. Grotheer, In TRANSPORT PHENOMENA IN COMBUSTION. Transport phenomena in combustion International symposium; 8th, Transport phenomena in combustion; Washington, D., Ed.; Taylor \&Francis (1996) 130-141.

[10] K. Hoyermann, J. Seeba, Proc. Combust. Inst. 25 (1994) 851-858.

[11] H.H. Nelson, J.R. McDonald, J. Phys. Chem. 86 (1982) 1242-1244.

[12] G. da Silva, J.W. Bozzelli, J. Phys. Chem. A 112 (2008) 3566-3575.

[13] Y. Georgievskii, S.J. Klippenstein, J. Phys. Chem. A 107 (2003) 9776-9781.

[14] V.V. Kislov, R.I. Singh, D.E. Edwards, A.M. Mebel, M. Frenklach, Proc. Combust. Inst. 35 (2015) 1861-1869.

[15] C.F. Goldsmith, L.B. Harding, Y. Georgievskii, J.A. Miller, S.J. Klippenstein, J. Phys. Chem. A 119 (2015) 7766-7779.

[16] C.P. Moradi, A.M. Morrison, S.J. Klippenstein, C.F. Goldsmith, G.E. Douberly, J. Phys. Chem. A 117 (2013) 13626-13635.

[17] S.J. Klippenstein, J. Chem. Phys. 96 (1992) 367-371.

[18] S.J. Klippenstein, Y. Georgievskii, L.B. Harding, Phys. Chem. Chem. Phys. 8 (2006) 1133-1147.

[19] Y. Georgievskii, S.J. Klippenstein, J. Chem. Phys. 122 (2005) 194103.

[20] Y. Georgievskii, L. B. Harding, S. J. Klippenstein,; http://tcg.cse.anl.gov/papr/codes/varecof.html.

[21] K. Andersson, P.Å. Malmqvist, B.O. Roos, J. Chem. Phys., 96 (1992) 1218-1226.

[22] G. Ghigo, B.O. Roos, P.-Å. Malmqvist, Chem. Phys. Lett. 396 (2004) 142-149.

[23] J.M.L. Martin, O. Uzan, Chem. Phys. Lett. 282 (1998) 16-24.

[24] G. Knizia, T.B. Adler, H.-J. Werner, J. Chem. Phys. 130 (2009) 054104.

[25] T.B. Adler, G. Knizia, H.-J. Werner, J. Chem. Phys. 127 (2007) 221106.

[26] F.R. Manby, H.-J. Werner, T.B. Adler, A.J. May, J. Chem. Phys. 124 (2006) 094103.

[27] L. Goerigk, S. Grimme, Phys. Chem. Chem. Phys. 13 (2011) 6670-6688.

[28] M.J. Frisch, G.W. Trucks, H.B. Schlegel, G.E. Scuseria, M.A. Robb, Gaussian 09, Revision B.01, Gaussian Inc., Wallingford, CT (2009).

[29] H.J. Werner, P.J. Knowles, G. Knizia, F.R. Manby, M. Schutz, MOLPRO, version 2010.1; www.molpro.net.

[30] Y. Murakami, T. Oguchi, K. Hashimoto, Y. Nosaka, J. Phys. Chem. A 111 (2007) 13200-13208.

[31] S.J. Klippenstein, Proc. Comb. Inst. 36, in press (2016).

[32] K. Tonokura, Y. Norikane, M. Koshi, Y. Nakano, S. Nakamichi, M. Goto, S. Hashimoto, M. Kawasaki, M. Sulbaek Andersen, M. Hurley, J. Phys. Chem. A 106 (2002) 5908-5917. 
[33] K. Tanaka, M. Ando, Y. Sakamoto, K. Tonokura, Int. J. Chem. Kinet. 44 (2012) 41-50.

[34] L. Elmaimouni, R. Minetti, J.P. Sawerysyn, P. Devolder, Int. J. Chem. Kinet. 25 (1993) 399-413.

[35] T. Ebata, K. Obi, I. Tanaka, Chem. Phys. Lett. 77 (1981) 480-483. 
Figure Captions

Fig. 1 Optimized ROO complexes and reaction enthalpies at $0 \mathrm{~K}(\mathrm{kcal} / \mathrm{mol})$ for (a) phenyl, (b) benzyl, (c) 1-naphthyl, and (d) 2-naphthyl radicals.

Fig. 2 MEP energies for the phenyl $+\mathrm{O}_{2}$ reaction from different corrections. PT2 refers to CASPT2, CI refers to MRCI, while M1 represents the method correction evaluated from Eq. (E1).

Fig. 3 VRC-TST predicted recombination rate constants for phenyl $+\mathrm{O}_{2}$.

Fig. 4 Comparison between our best prediction and previously reported rate constants for phenyl + $\mathrm{O}_{2}$ recombination.

Fig. 5 MEP for $1-\mathrm{C}_{10} \mathrm{H}_{7}+\mathrm{O}_{2}$ and $2-\mathrm{C}_{10} \mathrm{H}_{7}+\mathrm{O}_{2}$.

Fig. 6 Comparison between VRC-TST rate constants and previous experimental and theoretical studies for $\mathrm{C}_{6} \mathrm{H}_{5}+\mathrm{O}_{2}$ (black), 1- $\mathrm{C}_{10} \mathrm{H}_{7}+\mathrm{O}_{2}$ (red), and 2- $\mathrm{C}_{10} \mathrm{H}_{7}+\mathrm{O}_{2}$ (blue).

Fig. 7 MEP curves for benzyl $+\mathrm{O}_{2}$ at various levels. PT2(75) refers to the CASPT2(7e,5o) method, PT2(1311) refers to the CASPT2(13e,11o) method, and (CI+Q) refers to MRCI+Q(7e,5o) energies.

Fig. 8 Comparison of the VRC-TST predicted recombination rate constants and previous experimental measurements for benzyl $+\mathrm{O}_{2}$. 